\title{
PARALLEL CONNECTIONS OVER SYMMETRIC SPACES
}

\author{
Luis GuiJarro, Lorenzo Sadun, and Gerard Walschap
}

\begin{abstract}
Let $M$ be a simply connected Riemannian symmetric space, with at most one flat direction. We show that every Riemannian (or unitary) vector bundle with parallel curvature over $M$ is an associated vector bundle of a canonical principal bundle, with the connection inherited from the principal bundle. The problem of finding Riemannian (or unitary) vector bundles with parallel curvature then reduces to finding representations of the structure group of the canonical principal bundle.
\end{abstract}

This paper concerns connections on Riemannian vector bundles over simply connected symmetric spaces. Given a hypothesis about the Riemannian curvature of a symmetric space, we show that every Riemannian vector bundle with parallel curvature over that space is an associated vector bundle of a canonical principal bundle, with the connection inherited from the principal bundle. The problem of finding Riemannian vector bundles with parallel curvature then reduces to finding representations of the structure group of the canonical principal bundle. Our results apply also to unitary vector bundles, since a $\mathbb{C}^{k}$ bundle can be viewed as an $\mathbb{R}^{2 k}$ bundle together with an additional structure.

The hypothesis we put on a symmetric space is quite mild, and is satisfied by all simply connected irreducible symmetric spaces, and by simply connected reducible symmetric spaces that do not contain an $\mathbb{R}^{2}$ factor. If a symmetric space does contain such a factor, then the theorem does not hold, and we give explicit counterexamples.

Recall that a Riemannian connection on a vector bundle $E \rightarrow M$ is said to be Yang-Mills if its curvature tensor $R^{E}$ is harmonic; i.e., if $d_{A}^{*} R^{E}=0$, where $d_{A}^{*}$ is the covariant divergence operator. Yang-Mills connections have generated substantial interest, and much of our current knowledge of the topology of smooth 4-manifolds comes from their study [DK]. In the special case of the Levi-Civita connection on the tangent bundle of a Riemannian manifold, there is a stronger concept which has been of central importance in Riemannian geometry. A Riemannian manifold $M$, with Riemann curvature tensor $R^{M}$, is said to be locally symmetric if all covariant derivatives of $R^{M}$ vanish. This is equivalent to $R^{M}(X, Y) Z$ being a parallel vector field along every path $\gamma$ for any $X, Y, Z$ parallel along $\gamma$. This concept generalizes naturally to arbitrary Riemannian vector bundles $E \rightarrow M$ :

Definition. A connection on $E$ is said to be parallel if $\nabla R^{E}=0$; equivalently, if for any smooth path $\gamma$ of $M$, parallel vector fields $X, Y$ along $\gamma$, and parallel section $U$ of $E$ along $\gamma$, the section $R^{E}(X, Y) U$ is parallel along $\gamma$. 
This condition involves, of course, the Riemannian metric on the base manifold $M$. (By a Riemannian vector bundle we mean a vector bundle with an inner product on each fiber. A connection on such a bundle is required to respect the inner product, meaning that the inner product is parallel. In this paper all vector bundles are assumed to be real and Riemannian, except where stated otherwise.)

Recall that a symmetric space is a Riemannian manifold $M$ for which the geodesic symmetry at any point is a global isometry of $M$. Every locally symmetric space has a globally symmetric universal cover. Conversely, every symmetric space is locally symmetric. If $M$ is a symmetric space, then $M$ can be written as $G_{0} / H_{0}$, where $G_{0}$ and $H_{0}$ are groups, with $H_{0}=\operatorname{Hol}(M)$, the holonomy group of $M$. For example, $S^{n}=S O(n+1) / S O(n)$ and $\mathbb{C P}^{n}=[U(n+1) / U(1)] / U(n)$. By lifting to the universal cover of $G_{0}$ we can write $M=G / H$, where $G$ is simply connected and $H$ is a cover of $H_{0}$. If $M$ is simply connected, then $H$ is connected. For example, $S^{n}=\operatorname{Spin}(n+1) / \operatorname{Spin}(n)$ and $\mathbb{C P}^{n}=S U(n+1) / U(n)$. (In the $\mathbb{C P}^{n}$ example, $H_{0}$ and $H$ are both isomorphic to $U(n)$ as groups, but $H$ is still the $n+1$-fold cover of $H_{0}$.)

Let $M_{p}$ denote the tangent space of $M$ at $p$. The space $\Lambda^{2}\left(M_{p}\right)$ is naturally identified with $\mathfrak{s o}\left(M_{p}\right)$, the Lie algebra of skew-adjoint endomorphisms of $M_{p}$, by requiring that

$$
u \wedge v(w)=\langle u, w\rangle v-\langle v, w\rangle u, \quad u, v, w \in M_{p}
$$

The holonomy of $M$ acts on $\Lambda^{2}$ in a natural way via $h(u \wedge v)=h u \wedge h v$, for $h \in \operatorname{Hol}(M)$. Under the above identification, the action of Hol is by conjugation on $\mathfrak{s o}\left(M_{p}\right)$. This is also true for $\Lambda^{2} E$ and the holonomy group in any vector bundle $E$ with a Riemannian connection. Furthermore, the Riemannian curvature $R^{M}$ at $p$ is an equivariant map from $\mathfrak{s o}\left(M_{p}\right)$ to itself. It is easy to see that in general $\left[\operatorname{ker} R^{M}, \operatorname{Im} R^{M}\right] \subset \operatorname{ker} R^{M}$, and therefore the same is valid for the linear subspace spanned by $\left[\operatorname{ker} R^{M}, \operatorname{Im} R^{M}\right.$ ]. For our purposes, we will consistently assume that $R^{M}$ satisfies a stronger condition:

Condition A: $\operatorname{span}\left[\operatorname{ker} R^{M}, \operatorname{Im} R^{M}\right]=\operatorname{ker} R^{M}$.

This condition is well understood; in fact, in $\S 4$ we will prove:

Theorem 1.1. Let $M$ be a simply connected Riemannian symmetric space. Condition $A$ holds unless $M$ is the product of $\mathbb{R}^{2}$ and another symmetric space, in which case Condition A fails.

Put another way, Condition A holds as long as the dimension of the center of $G$ does not exceed 1. This is discussed in detail in section 4 .

Since the Levi-Civita connection on the tangent bundle of a symmetric space $M$ is parallel, any bundle built from the tangent bundle will also admit a parallel connection, naturally induced from the Levi-Civita connection on $T M$. In particular ${ }_{k}^{\otimes} T M$ and $\Lambda^{k}(T M)$ admit parallel connections, as does the (principal) bundle $\operatorname{Fr}(T M)$ of orthonormal frames of $T M$. Sub-bundles of $\operatorname{Fr}(T M)$, and finite covers of $\operatorname{Fr}(T M)$ or of sub-bundles of $\operatorname{Fr}(T M)$, also inherit parallel connections, whenever such subbundles are invariant by parallel transport.

If $B$ is a principal $H$-bundle over $M$ and $\rho: H \rightarrow S O(k)$ is a representation of 
equivalence $(b h, v) \sim(b, \rho(h) v)$. The equivalence class of $(b, v)$ is denoted $[(b, v)]$. The vector bundle $E$ inherits a connection from that of $B$, which will be parallel if the connection on $B$ is parallel. Thus vector bundles with parallel connections naturally arise from representations of the holonomy group of a symmetric space $M$, or of its finite covers.

Parallel connections are also related to group actions. Let $G$ be a group that acts orthogonally and transitively on a vector bundle $E$, covering a group of isometries of $M$, and suppose that $E$ has a connection that is invariant under this $G$-action. Then that connection is known to be parallel. Furthermore, for any fixed bundle $E$ and $G$-action, Wang's theorem [W] classifies the $G$-invariant connections.

In this paper we show that, on symmetric spaces satisfying Condition A, these two constructions are equivalent, and are the only source of parallel connections:

Main Theorem. Let $M=G / H$ be a simply connected symmetric space written as a canonical group quotient, with $G$ simply connected, and suppose that the canonical metric on $M$ satisfies Condition $A$. Let $E$ be any rank- $k$ real vector bundle with parallel connection over $M$. Then there exists a representation $\rho: H \rightarrow S O(k)$ such that $E$ is isomorphic to the vector bundle $E^{\prime}=G \times{ }_{\rho} \mathbb{R}^{k}$, with the isomorphism taking the natural connection on $E^{\prime}$ to the given connection on $E$.

Corollary 1. Every parallel connection over $M$ is invariant under a $G$-action on the bundle that covers the natural $G$-action on $M$.

Corollary 2. Every unitary rank- $k$ vector bundle with parallel connection over $M$ is isomorphic to $G \times{\rho^{\prime}} \mathbb{C}^{k}$, where $\rho^{\prime}: H \rightarrow U(k)$ is a rank-k unitary representation of $H$.

Several authors have investigated the question of when the action of a given transitive group of isometries on the base $M$ can be lifted to a bundle over $M$, see for instance $[\mathrm{B}-\mathrm{H}]$. Since such a lifted action implies the existence of a parallel connection, Corollary 1 provides a complete answer: Over a symmetric space satisfying the hypotheses of the main theorem, the vector bundles that admit lifts of transitive group actions are exactly the bundles that admit parallel connections, which are exactly the associated vector bundles of $G$ itself. Notice, though, that $G$ is usually a cover of the isometry group $G_{0}$ of the base, and that the action of the latter will not, in general, lift to the bundle: For example, the action of $S O(5)$ on the 4-sphere does not lift to the spinor bundle, even though that of $\operatorname{Spin}(5)$ does.

The main theorem tells us that a local condition on a bundle with connection, namely that its curvature be parallel, implies a rigid global structure. However, for this to be true we need assumptions about the underlying manifold, namely that it be a simply connected symmetric space whose metric connection satisfies Condition A. Without these conditions the conclusions of the theorem can be false, as the following counterexamples show.

Let $M=T^{2}=G / H$, where $G=\mathbb{R}^{2}$ and $H=\mathbb{Z}^{2} . M$ is not simply connected and Condition A fails, as $\operatorname{ker} R^{M}$ is all of $\Lambda^{2}\left(M_{p}\right)$ while $\operatorname{Im} R^{M}$ is zero. Since $R^{M}=0$, any bundle constructed from a representation of $H$ is necessarily flat (although possibly with nontrivial holonomy). However, there exist bundles and connections over $T^{2}$ with parallel nonzero curvature. For example, consider the 
$f(x+n, y+m)=\exp (2 \pi i n y) f(x, y)$ gives a complex line bundle of Chern class +1 over $T^{2}$ that we denote $E$. $E$ admits many connections of constant nonzero curvature; one has connection form $A=-2 \pi i x d y$. Not only does this line bundle not come from a representation of $H$, but the connection is not invariant under translation. Indeed, the curvature itself measures the extent to which holonomy changes when a homologically nontrivial loop is translated. There is a 2-parameter family of parallel connections on $E$, indexed by $T^{2}$ itself, and translation takes one such connection into another.

Now let $M=G=\mathbb{R}^{2}$, with $H$ trivial. $M$ is simply connected but does not satisfy Condition A. Once again, any bundle built from the tangent bundle with its canonical structure has a flat connection, but there are connections over $\mathbb{R}^{2}$ that are parallel but not flat. For example, one can again look at a complex line bundle with connection form $A=-2 \pi i x d y$ and constant curvature $-2 \pi i d x \wedge d y$. Unlike the $T^{2}$ example, this connection is invariant under translation, so Wang's theorem does apply.

The proof of the main theorem is based on the following observations. Let $M=G / H$ be a simply connected symmetric space, with $G$ simply connected. $H$ is a covering space of $\operatorname{Hol}(M)$, the holonomy group of $M$. Let $\pi: H \rightarrow \operatorname{Hol}(M)$ be the covering map, so that $T M=G \times{ }_{\pi} \mathbb{R}^{n}$. Suppose $E=G \times{ }_{\rho} R^{k}$, for a representation $\rho: H \rightarrow S O(k)$, and endow $E$ with the connection induced by the one on $G$. Then the holonomy around a loop $\gamma$ for the three bundles $T M, G$, and $E$ are related by the commutative diagram

$$
\begin{aligned}
\gamma & \\
\mathbb{P}_{\gamma}^{M} \swarrow & \downarrow \mathbb{P}_{\gamma}^{G} \searrow \mathbb{P}_{\gamma}^{E} \\
\operatorname{Hol}\left(M_{p}\right) \stackrel{\pi}{\longleftarrow} & H \stackrel{\rho}{\longrightarrow} \operatorname{Hol}\left(E_{p}\right),
\end{aligned}
$$

where $\mathbb{P}_{\gamma}^{E}$ denotes parallel translation in $E$ along the curve $\gamma$ in $M$, etc. One cannot compute $\mathbb{P}_{\gamma}^{E}$ directly from $\mathbb{P}_{\gamma}^{T M}$, since $\pi$ is not $1-1$. However, looking at infinitesimal loops we have the commutative diagram

$$
\begin{gathered}
\Lambda^{2}\left(M_{p}\right) \\
R^{M} \swarrow \quad \downarrow R^{G} \searrow R^{E} \\
\mathfrak{h o l}\left(M_{p}\right) \stackrel{\hat{\pi}}{\longleftarrow} \mathfrak{h} \stackrel{\hat{\rho}}{\longrightarrow} \mathfrak{h o l}\left(E_{p}\right),
\end{gathered}
$$

where $\hat{\pi}: \mathfrak{h} \rightarrow \mathfrak{s o}\left(M_{p}\right)$ and $\hat{\rho}: \mathfrak{h} \rightarrow \mathfrak{s o}\left(E_{p}\right)$ are the Lie algebra homomorphisms corresponding to the group homomorphisms $\pi$ and $\rho$. Since $\pi$ is a covering map, $\hat{\pi}$ is an algebra isomorphism, and we have

$$
R^{E}=\hat{\rho} \circ(\hat{\pi})^{-1} \circ R^{M}
$$

Since $R^{M}$ is parallel and $\hat{\rho}$ and $\hat{\pi}$ are fixed, $R^{E}$ is parallel. Notice that $\operatorname{ker} R^{M} \subset$ ker $R^{E}$, so that $R^{E} \circ\left(R^{M}\right)^{-1}$ is well-defined (although $\left(R^{M}\right)^{-1}$ may not be), and is a Lie algebra homomorphism from $\mathfrak{h o l}\left(M_{p}\right)$ to $\mathfrak{h o l}\left(E_{p}\right)$. Indeed, it is equal to $\hat{\rho} \circ \hat{\pi}^{-1}$.

Now suppose that $E$ is a vector bundle over $M=G / H$ with parallel curvature. 
First we show that ker $R^{M} \subset \operatorname{ker} R^{E}$. This is the step where condition A is needed. Then, with $\mathfrak{h}$ identified with $\mathfrak{h o l}\left(M_{p}\right)$, we define $\hat{\rho}=R^{E} \circ\left(R^{M}\right)^{-1}$ and show that it is a Lie algebra homomorphism from $\mathfrak{h}=\mathfrak{h o l}\left(M_{p}\right)$ to $\mathfrak{h o l}\left(E_{p}\right)$. Next, we exponentiate this algebra homomorphism to get a group homomorphism $\rho: H \rightarrow \operatorname{Hol}\left(E_{p}\right) \subset$ $S O(k)$. This is somewhat delicate, since $H$ may not be simply connected. To get around this, we extend $\hat{\rho}$ to an algebra homomorphism from $\mathfrak{g}$ to the Lie algebra of the extended gauge group, exponentiate that to a group homomorphism from $G$ to the extended gauge group, and then restrict the group homomorphism to $H$. This is the step that requires the simple connectivity of $G$.

Having reconstructed $\rho$ from the curvature of $E$, we define $E^{\prime}=G \times{ }_{\rho} \mathbb{R}^{k}$. Identifying the fibers of $E$ and $E^{\prime}$ at our base point $p$, we note that the curvatures of $E$ and $E^{\prime}$ are equal, and indeed the two vector bundles have the same holonomy around any given loop. We then use parallel transport to identify the fibers of $E$ and $E^{\prime}$ at every point, completing the proof.

In $\S 2$ we establish the local properties of $R^{M}$ and $R^{E}$, where $E$ is any vector bundle with parallel curvature. In particular, we show (Theorem 2.4) that $R^{E}$ o $\left(R^{M}\right)^{-1}$ is a Lie algebra homomorphism. In $\S 3$ we construct the bundle $E^{\prime}$ and show that it is isomorphic to $E$. In $\S 4$ we consider Condition $A$, and show that it holds on all simply connected symmetric spaces that do not have an $\mathbb{R}^{2}$ factor. Finally, in $\S 5$ we discuss some of the implications of the Main Theorem.

\section{LOCAL PROPERTIES OF PARALLEL CONNECTIONS}

We briefly recall some basic facts about holonomy and curvature in bundles. The reader is referred to $[\mathrm{P}]$ for further details and other facts that will be freely used here. It will often be convenient to work with principal bundles instead of vector bundles. Recall that an oriented Riemannian vector bundle $E$ of $\operatorname{rank} k$ over $M$ is isomorphic to $\operatorname{Fr}(E) \times{ }_{\rho_{0}} \mathbb{R}^{k}$, where $\operatorname{Fr}(E)$ is the principal $S O(k)$ bundle of oriented orthonormal frames of $E$ and $\rho_{0}$ is the fundamental representation of $S O(k)$. A sub-bundle of $\operatorname{Fr}(E)$ is obtained by taking all parallel translates of a given frame. This is a principal bundle over $M$, whose fiber is the holonomy group $\operatorname{Hol}(E)$ of $E$.

A connection on $E$ induces one on the corresponding principal bundle $\operatorname{Fr}(E)$, on all sub-bundles of $\operatorname{Fr}(E)$, and on all covers of sub-bundles of $\operatorname{Fr}(E)$. Conversely, if $B$ is a principal $H$-bundle over $M$ and $\rho$ is an orthogonal $k$ dimensional representation of $H$, then a connection on $B$ induces one on the vector bundle $E=B \times{ }_{\rho} \mathbb{R}^{k}$, which will be parallel if the connection on $B$ is parallel.

The curvature tensor $R^{E}$ of a connection on $E$ is a bundle map $\Lambda^{2} T M \rightarrow$ $\mathfrak{h o l}(E) \subset \mathfrak{s o}(E)$. A result of Ambrose and Singer says that the fiber of $\mathfrak{h o l}(E)$ over $p \in M$ is spanned by the parallel translates of all curvature transformations $R^{E}(x \wedge y), x \wedge y \in \Lambda^{2} M_{q}, q \in M$, along smooth curves from $q$ to $p[\mathrm{P}]$. When the connection is parallel, the curvature is invariant under parallel translation, and we obtain:

Lemma 2.1. Let $E$ be a vector bundle with parallel connection over a manifold $M$, and let $p$ be a point on $M$, then

(1) $R^{E}: \Lambda^{2}\left(M_{p}\right) \rightarrow \mathfrak{h o l}\left(E_{p}\right)$ is onto. 
then

$R^{E} \circ \mathbb{P}_{\gamma}^{\mathfrak{s o}(T M)}(A)=R^{E}\left(\mathbb{P}_{\gamma}^{T M} A \mathbb{P}_{-\gamma}^{T M}\right)=\mathbb{P}_{\gamma}^{E} R^{E}(A) \mathbb{P}_{-\gamma}^{E}=\mathbb{P}_{\gamma}^{\mathfrak{s o}(E)} \circ R^{E}(A)$

(3) For $A, B \in \Lambda^{2}\left(M_{p}\right), R^{E}\left[R^{M} A, B\right]=\left[R^{E} A, R^{E} B\right]$.

Proof. (1) and (2) are clear from the above discussion, together with the fact that the holonomy acts by conjugation on both $\mathfrak{s o}(T M)$ and $\mathfrak{s o}(E)$. It suffices to establish (3) for decomposable elements $A=x \wedge y, B=z \wedge w, x, y, z, w \in M_{p}$. So consider vector fields $X, Y$ on $M$ such that $X_{p}=x, Y_{p}=y$, and $[X, Y]=0$ in a neighborhood of $p$. If $\left\{\phi_{s}\right\}$ and $\left\{\psi_{s}\right\}$ denote the local 1-parameter groups of $X$ and $Y$, then for small $t$, the product $\gamma_{t}$ of the curves $s \mapsto \phi_{\sqrt{s}}(p), s \mapsto \psi_{\sqrt{s}} \phi_{\sqrt{t}}(p)$, $s \mapsto \phi_{-\sqrt{s}} \psi_{\sqrt{t}} \phi_{\sqrt{t}}(p)$, and $s \mapsto \psi_{-\sqrt{s}} \phi_{-\sqrt{t}} \psi_{\sqrt{t}} \phi_{\sqrt{t}}(p), 0 \leq s \leq t$, is a piecewise smooth loop at $p$, such that

$$
\left.\frac{d}{d t}\right|_{0} \mathbb{P}_{\gamma_{t}}^{T M}=R^{M}(A), \quad \text { and }\left.\frac{d}{d t}\right|_{0} \mathbb{P}_{\gamma_{t}}^{E}=R^{E}(A)
$$

cf. also [P]. If $\beta$ denotes the curve $t \mapsto R^{E} \mathbb{P}_{\gamma_{t}}^{\mathfrak{s o}(T M)}(B)$ in $\Lambda^{2}\left(M_{p}\right)$, we have that $\beta(t)=R^{E} \mathbb{P}_{\gamma_{t}}^{T M} B\left(\mathbb{P}_{\gamma_{t}}^{T M}\right)^{-1}$, and by the first identity in $(2.2), \dot{\beta}(0)=R^{E}\left[R^{M} A, B\right]$. On the other hand, $\beta(t)=\mathbb{P}_{\gamma_{t}}^{\mathfrak{s o}(E)} R^{E}(B)=\mathbb{P}_{\gamma_{t}}^{E} R^{E}(B)\left(\mathbb{P}_{\gamma_{t}}^{E}\right)^{-1}$, and the second identity in $(2.2)$ yields $\dot{\beta}(0)=\left[R^{E}(A), R^{E}(B)\right]$, which establishes the claim.

We now consider the case when the base is a locally symmetric space. The curvature tensor of the Levi-Civita connection then satisfies the following property:

Proposition 2.2. Let $M$ be a locally symmetric space, $E_{\lambda} \subset \mathfrak{s o}\left(M_{p}\right)$ the $\lambda$ eigenspace of the curvature operator $R^{M}: \mathfrak{s o}\left(M_{p}\right) \rightarrow \mathfrak{s o}\left(M_{p}\right)$. If $\lambda \neq 0$, then $E_{\lambda}$ is an ideal of the algebra bundle $\mathfrak{h o l}\left(M_{p}\right) \subset \mathfrak{s o}\left(M_{p}\right)$. Thus, hol $\left(M_{p}\right)$ is a Whitney sum $\oplus E_{i}$ of parallel eigenspace bundles, which are fiberwise ideals.

Remark. Adding the 0-eigenbundle of the curvature decomposes $\mathfrak{s o}(T M)$ as a Whitney sum of parallel subbundles. In general, however, the kernel of $R^{M}$ is not closed under the Lie bracket.

Proof of Proposition 2.2. Let $A$ and $B$ denote $\lambda$-eigenvectors. Then Lemma 2.1(3) with $E=T M$ implies

$$
\lambda R^{M}[A, B]=R^{M}\left[R^{M} A, B\right]=\left[R^{M} A, R^{M} B\right]=\lambda^{2}[A, B],
$$

so that $E_{\lambda}$ is a subbalgebra bundle if $\lambda \neq 0$. Similarly, for nonzero $\mu \neq \lambda$, and $\lambda$-eigenvector $A$, $\mu$-eigenvector $B$, we have

$$
\begin{aligned}
\lambda R^{M}[A, B]=R^{M}\left[R^{M} A, B\right] & =\left[R^{M} A, R^{M} B\right] \\
& =R^{M}\left[A, R^{M} B\right]=\mu R^{M}[A, B],
\end{aligned}
$$

where the identity $\left[R^{M} A, R^{M} B\right]=R^{M}\left[A, R^{M} B\right]$ follows by interchanging $A$ and $B$ in Lemma 2.1(3). Thus, $[A, B] \in \operatorname{ker} R^{M} \cap \mathfrak{h o l}\left(M_{p}\right)=\{0\}$. 
Lemma 2.3. If $M$ is a locally symmetric space satisfying Condition $A$ and $R^{E}$ is the curvature of a parallel connection on a bundle $E$ over $M$, then $\operatorname{ker} R^{M} \subset \operatorname{ker} R^{E}$.

Proof. Suppose that $A \in E_{\lambda}$ and that $B \in \operatorname{ker} R^{M}$, with $\lambda \neq 0$. Then

$$
\lambda R^{E}[A, B]=R^{E}\left[R^{M} A, B\right]=\left[R^{E} A, R^{E} B\right]=R^{E}\left[A, R^{M} B\right]=0 .
$$

Thus $R^{E}\left[E_{\lambda}, \operatorname{ker} R^{M}\right]=0$. Summing over $\lambda$ we have that $R^{E}\left[\operatorname{Im} R^{M}, \operatorname{ker} R^{M}\right]=0$. But by Condition $\mathrm{A}, \operatorname{span}\left[\operatorname{Im} R^{M}, \operatorname{ker} R^{M}\right]=\operatorname{ker} R^{M}$, so $R^{E}\left(\operatorname{ker} R^{M}\right)=0$.

Since $R^{M}$ is onto $\mathfrak{h o l}\left(M_{p}\right)$, and $R^{E}\left(\operatorname{ker} R^{M}\right)=0$, there exists a map $\hat{\rho}: \mathfrak{h o r}\left(M_{p}\right) \rightarrow$ $\mathfrak{s o}\left(E_{p}\right)$ such that $R^{E}=\hat{\rho} \circ R^{M}$. Formally we write $\hat{\rho}=R^{E} \circ\left(R^{M}\right)^{-1}$.

Theorem 2.4. Let $M$ denote a symmetric space, $R^{E}$ the curvature at a point $p$ of a parallel connection on a bundle $E$ over $M$. Then $\hat{\rho}:=R^{E} \circ\left(R^{M}\right)^{-1}: \mathfrak{h o l}\left(M_{p}\right) \rightarrow$ $\mathfrak{s o}\left(E_{p}\right)$ is a Lie algebra bundle homomorphism whose image is all of $\mathfrak{h o l}\left(E_{p}\right)$.

Proof. Since $R^{E}=\hat{\rho} \circ R^{M}$, the image of $\hat{\rho}$ is the image of $R^{E}$, namely $\mathfrak{h o l}\left(E_{p}\right)$. To see that $\hat{\rho}$ is a Lie algebra homomorphism, we need to check that $\hat{\rho}[A, B]=$ $[\hat{\rho}(A), \hat{\rho}(B)]$ when $A$ and $B$ belong to a common eigenspace $E_{\lambda}$ of $R^{M}$, and when $A \in E_{\mu}, B \in E_{\lambda}, \lambda \neq \mu$. Let $b=\frac{1}{\lambda} B \in\left(R^{M}\right)^{-1} B$. In the former case,

$$
[\hat{\rho}(A), \hat{\rho}(B)]=R^{E}[A, b]=\frac{1}{\lambda} R^{E}[A, B]=\hat{\rho}[A, B],
$$

since $[A, B]$ belongs to $E_{\lambda}$. In the latter case, we have

$$
[\hat{\rho}(A), \hat{\rho}(B)]=R^{E}[A, b]=\frac{1}{\lambda} R^{E}[A, B] .
$$

After interchanging the roles of $A$ and $B$, we see that the left side of $(2.7)$ is also equal to $(1 / \mu) R^{E}[A, B]$, and therefore vanishes. However, $[A, B]=0$ by Proposition 2.2 , so $[\hat{\rho}(A), \hat{\rho}(B)]=0=\hat{\rho}[A, B]$.

\section{The global Structure of $E$}

In this section we complete the proof of the main theorem, using the local structure of $\S 2$ to construct a bundle $E^{\prime}$, and then showing that $E$ and $E^{\prime}$ are isomorphic with the isomorphism respecting the connections. Our base manifold is a Riemannian symmetric space $M$. Recall that $M=G_{0} / H_{0}$, where $G_{0}$ is the largest connected group of isometries of $M$, and $H_{0}$ is the isotropy group at some fixed point $p$. If $\lambda: H_{0} \rightarrow S O\left(M_{p}\right)$ denotes the (faithful) linear isotropy representation $\lambda(h)=h_{* p}$, then the tangent bundle $T M$ is isomorphic to $G_{0} \times_{\lambda} M_{p}$ via $[(g, u)] \mapsto g_{* p} u$, and the holonomy group of $M$ at $p$ is $\lambda\left(H_{0}\right)$. An equivalent description of the tangent bundle is obtained by considering the involutive automorphism $\sigma$ of $G_{0}$ given by $\sigma(g)=s_{p} \circ g \circ s_{p}^{-1}$, where $s_{p}$ denotes the geodesic symmetry at $p$. Its derivative at the identity squares to 1 and decomposes the Lie algebra $\mathfrak{g}$ into a sum $\mathfrak{g}=\mathfrak{h} \oplus \mathfrak{m}$ of the plus and minus 1 eigenspaces of $\sigma_{* e}$. The derivative of the projection $\pi: G_{0} \rightarrow M$ (which sends $g$ to $g(p)$ ) has kernel $\mathfrak{h}$ and thus identifies $\mathfrak{m}$ with the tangent space of $M$ at $p$. A straightforward computation shows that for $h \in H_{0}$, the diagram

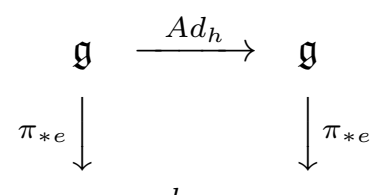


commutes, so that $T M$ is also identified with $G_{0} \times_{A d} \mathfrak{m}$, with $A d$ denoting the adjoint representation of $H_{0}$.

We work throughout with $M=G / H$ where $G$ is the universal cover of $G_{0}$, and identify the Lie algebra $\mathfrak{h}$ of $H$ with $\mathfrak{h o l}\left(M_{p}\right)$. Our first task is to promote the Lie algebra homomorphism $\hat{\rho}: \mathfrak{h o l}\left(M_{p}\right) \rightarrow \mathfrak{s o}\left(E_{p}\right)$ into a group homomorphism $\rho: H \rightarrow S O\left(E_{p}\right)$. Since in general $H$ is not simply connected, this promotion is not automatic. We must extend $\hat{\rho}$ to a homomorphism from $\mathfrak{g}$ to an appropriate Lie algebra, promote that extension to a group homomorphism, and then restrict the homomorphism to $H$.

Recall that if $B$ is a principal $K$-bundle over $M$, the gauge group $\mathcal{G}_{B}$ of $B$ is the group of $K$-equivariant diffeomorphisms of $B$ that cover the identity on $M$, cf. e.g. [BL]. It is canonically identified with the space of sections of the bundle of groups $B \times{ }_{c} K$, where $c$ denotes conjugation; i.e., $\left(b k_{1}, k_{2}\right) \sim\left(b, k_{1} k_{2} k_{1}^{-1}\right)$. The gauge algebra $\mathfrak{G}_{B}$ of $B$ is similarly defined as the Lie algebra of sections of the Lie algebra bundle $B \times{ }_{\text {Ad }} \mathfrak{K}$, and there is a fiberwise exponential map

$$
\exp : \mathfrak{G}_{B} \rightarrow \mathcal{G}_{B}
$$

In the case of a Riemannian vector bundle $E$, the gauge group $\mathcal{G}_{E}$ and algebra $\mathfrak{G}_{E}$ are defined to be the gauge group and algebra of the principal bundle $\operatorname{Fr}(E)$ of oriented orthonormal frames. Equivalently, $\mathcal{G}_{E}$ is the group of orthogonal bundle maps $E \rightarrow E$ that cover the identity on $M$. An element of $\mathfrak{G}_{E}$ can also be viewed as a (vertical) vector field on $E$.

Let $M=G / H$ be a symmetric space with $G$ simply connected, and $E$ a vector bundle over $M$. Define the enlarged gauge group of $E$ to be the group $\tilde{\mathcal{G}}_{E}$ of all orthogonal bundle maps $E \rightarrow E$ that cover the natural action of $G$ on $M$. The projection $\alpha: E \rightarrow M$ determines an exact sequence

$$
1 \rightarrow \mathcal{G}_{E} \rightarrow \tilde{\mathcal{G}}_{E} \stackrel{\alpha}{\longrightarrow} G \rightarrow 1
$$

which, when differentiated at the identity, yields the exact sequence of vector spaces

$$
0 \rightarrow \mathfrak{G}_{E} \rightarrow \tilde{\mathfrak{G}}_{E} \stackrel{\alpha_{*}}{\rightarrow} \mathfrak{g} \rightarrow 0
$$

A connection on $E$ determines a splitting of the sequence (3.4). An element $X \in$ $\tilde{\mathfrak{G}}_{E}$, viewed as a vector field on $E$, splits accordingly as $X=X^{h}+X^{v}$, where $X^{v}$ is a section of $\mathfrak{h o l}(E)$, and $\alpha_{*} X^{h}=\alpha_{*} X \in \mathfrak{g}$.

When $E=T M$, any $X \in \mathfrak{g}$ induces a vector field $\tilde{X} \in \tilde{\mathfrak{G}}_{M}$ on $T M$ : Identify $X$ with the Killing field on $M$ induced by the $G$-action. If $\phi_{t}$ denotes the oneparameter group generated by $X$, then $\phi_{t *}$ is a bundle map that covers $\phi_{t}$, and we set $\tilde{X}:=\left.\frac{d}{d t}\right|_{0}\left(\phi_{t *}\right)$. By standard properties of connections (see e.g. $\left.[\mathrm{P}]\right)$, one has

$$
\left[\tilde{X}^{v}, \tilde{Y}^{h}\right]=0, \quad\left[\tilde{X}^{h}, \tilde{Y}^{h}\right]^{v}=R^{M}(X, Y), \quad X, Y \in \mathfrak{g}
$$

Define a map $\phi: \mathfrak{g} \rightarrow \tilde{\mathfrak{G}}_{E}$ by $\phi(X)=X^{h}+\hat{\rho}\left(\tilde{X}^{v}\right)$, where $\hat{\rho}=R^{E} \circ\left(R^{M}\right)^{-1}$ : $\mathfrak{h o l}(T M) \rightarrow \mathfrak{h o l}(E)$ is the Lie algebra bundle homomorphism from Theorem 2.4, 
Lemma 3.1. $\phi: \mathfrak{g} \rightarrow \tilde{\mathfrak{G}}_{E}$ is a Lie algebra homomorphism.

Proof. We compute

$$
\begin{aligned}
\widetilde{[X, Y]} & =[\tilde{X}, \tilde{Y}]=[\tilde{X}, \tilde{Y}]^{h}+[\tilde{X}, \tilde{Y}]^{v}=\left[\tilde{X}^{h}, \tilde{Y}^{h}\right]^{h}+[\tilde{X}, \tilde{Y}]^{v} \\
& =\left[\tilde{X}^{h}, \tilde{Y}^{h}\right]^{h}+\left[\tilde{X}^{h}, \tilde{Y}^{h}\right]^{v}+\left[\tilde{X}^{v}, \tilde{Y}^{v}\right] \\
& =\left[\tilde{X}^{h}, \tilde{Y}^{h}\right]^{h}+R^{M}(X \wedge Y)+\left[\tilde{X}^{v}, \tilde{Y}^{v}\right] .
\end{aligned}
$$

Thus,

$$
\begin{aligned}
\phi[X, Y] & =\left[X^{h}, Y^{h}\right]^{h}+R^{E}(X \wedge Y)+\hat{\rho}\left[\tilde{X}^{v}, \tilde{Y}^{v}\right] \\
& =\left[X^{h}, Y^{h}\right]^{h}+R^{E}(X \wedge Y)+\left[\hat{\rho}\left(\tilde{X}^{v}\right), \hat{\rho}\left(\tilde{Y}^{v}\right)\right],
\end{aligned}
$$

since $\hat{\rho}$ is an algebra homomorphism. On the other hand,

$$
\begin{aligned}
{[\phi X, \phi Y] } & =\left[X^{h}+\hat{\rho}\left(\tilde{X}^{v}\right), Y^{h}+\hat{\rho}\left(\tilde{Y}^{v}\right)\right] \\
& =\left[X^{h}, Y^{h}\right]^{h}+\left[X^{h}, Y^{h}\right]^{v}+\left[\hat{\rho}\left(\tilde{X}^{v}\right), \hat{\rho}\left(\tilde{Y}^{v}\right)\right] \\
& =\left[X^{h}, Y^{h}\right]^{h}+R^{E}(X \wedge Y)+\left[\hat{\rho}\left(\tilde{X}^{v}\right), \hat{\rho}\left(\tilde{Y}^{v}\right)\right] .
\end{aligned}
$$

Proposition 3.2. Let $p$ denote the coset $e H \in G / H=M$. There exists a group homomorphism $\rho: H \rightarrow S O\left(E_{p}\right)$ whose derivative at the identity is $\left.\hat{\rho}\right|_{p}: \mathfrak{h} \rightarrow$ $\mathfrak{s o}\left(E_{p}\right)$.

Proof. Since $G$ is simply connected, the algebra homomorphism $\phi$ can be promoted to a group homomorphism $\Phi: G \rightarrow \tilde{\mathcal{G}}_{E}$. Restricting $\Phi$ to $H$ we get a map $H \rightarrow \tilde{\mathcal{G}}_{E}$. Now, if $X \in \mathfrak{h}$, then $X$, viewed as a Killing field on $M$, has a zero at $p$ because $H_{0}$ is the isotropy group of $p$. Therefore $\Phi(\exp (t X))$, and generally $\Phi(h)$ for any $h \in H$, preserves the fiber $E_{p}$. We define $\rho(h)$ to be the restriction of $\Phi(h)$ to that fiber. $\rho$ is a group homomorphism since $\Phi$ is, and the derivative of $\rho$ is $e v_{p} \circ \phi$, where $e v_{p}$ is evaluation at the base point $p$. But for $X \in \mathfrak{h}$,

$$
e v_{p} \circ \phi(X)=\tilde{X}^{h}(p)+\hat{\rho}\left(\tilde{X}^{v}\right)(p)=0+\hat{\rho}_{p}(X) .
$$

We now define the bundle $E^{\prime}$ to be $G \times{ }_{\rho} E_{p}$. $E^{\prime}$ inherits a natural parallel connection from $G$. The Main Theorem boils down to

Theorem 3.3. $E$ and $E^{\prime}$ are isomorphic vector bundles, with the isomorphism preserving the connection.

Proof. The fibers of $E$ and $E^{\prime}$ over $p$ are canonically identified. Denote the isomorphism $I_{p}: E_{p}^{\prime} \rightarrow E_{p}$. $I_{p}$ is an element of $\operatorname{Hom}\left(E^{\prime}, E\right)$. We must show that $I_{p}$ can be extended in a parallel fashion to all of $M$.

Let $F$ be the vector bundle $\operatorname{Hom}\left(E^{\prime}, E\right)$. $F$ inherits a parallel structure from those of $E^{\prime}$ and $E$. In particular, the holonomy of $F$ is generated by the curvature of $F$ at $p$, and this curvature is constructed from the curvatures of $E^{\prime}$ and $E$. By (1.4) the curvature of $E^{\prime}$ at $p$ is 
Thus, for any $A \in \Lambda^{2}\left(M_{p}\right), R^{E^{\prime}}(A)=R^{E}(A)$, and so

$$
R^{F}(A)\left(I_{p}\right)=0
$$

Since $I_{p}$ is in the kernel of $R^{F}(A)$ for every $A, \operatorname{Hol}(F)$ acts trivially on $I_{p}$, so $I_{p}$ can be extended, by parallel transport, to a parallel section of $F$. This section is our global isomorphism between $E^{\prime}$ and $E$.

This completes the proof of the main theorem. Corollary 1 is an obvious consequence. For Corollary 2, note that a rank- $k$ complex vector bundle is just a real rank- $2 k$ vector bundle with an additional feature, namely the complex structure $J$. If $E$ is a unitary bundle with parallel connection, then the holonomy of $E$ commutes with $J$, and so $\hat{\rho}_{p}$ commutes with $J$. Thus $\rho$ is actually a rank- $k$ unitary representation of $H$, not just a rank $2 k$ orthogonal representation.

In the proof of the Main Theorem we have constructed the representation $\rho$ as linear operators on the fixed vector space $E_{p}$ rather than as matrices acting on $\mathbb{R}^{k}$, and the definition of $E^{\prime}$ is similar. The difference between working with $E_{p}$ and $\mathbb{R}^{k}$ is of course nothing more than a choice of orthonormal basis for $E_{p}$.

\section{ThE CURVATURE CONDITION}

In this section we prove Theorem 1.1, showing that Condition A holds on all simply connected symmetric spaces that do not have an $\mathbb{R}^{2}$ factor. We begin with a special case:

Theorem 4.1. Condition A applies on all simply connected irreducible Riemannian symmetric spaces.

Proof. Let $M$ be such a space of dimension $n$, let $p \in M$ be a point, and suppose that $W:=\operatorname{span}\left[\operatorname{ker} R_{p}^{M}, \operatorname{Im} R_{p}^{M}\right] \subset \mathfrak{s o}\left(M_{p}\right)$ is a proper subspace of $V:=\operatorname{ker} R_{p}^{M}$. Let $A$ be an element of $V$, orthogonal to $W . A$ is then orthogonal to all of $\left[\mathfrak{s o}\left(M_{p}\right), \operatorname{Im} R^{M}\right]$, since $\left[\operatorname{Im} R^{M}, \operatorname{Im} R^{M}\right]$ is contained in $\operatorname{Im} R^{M}$, and so is orthogonal to $A$. Since Hol acts orthogonally on $\mathfrak{s o}\left(M_{p}\right), A$ is invariant under Hol.

Since $A$ is a skew endomorphism of $M_{p}$, it can be expressed as a block diagonal $n \times n$ matrix, where each block is either zero or a skew $2 \times 2$ matrix $L_{\lambda}=\left(\begin{array}{cc}0 & \lambda \\ -\lambda & 0\end{array}\right)$, with $\lambda>0$. For $\lambda \neq 0$, let $E_{\lambda} \subset M_{p}$ be the direct sum of the 2-planes with block $L_{\lambda}$. Since $A$ is invariant under Hol, so is $E_{\lambda}$, and so is the orthogonal complement of $E_{\lambda}$.

By de Rham's holonomy theorem (see e.g. [B]), $M$ is then the product of spaces $M_{1}$ and $M_{2}$ with tangent spaces $E_{\lambda}$ and $E_{\lambda}^{\perp}$. Since $M$ is irreducible, one of these spaces must be trivial. Since $A \neq 0$, we can find a $\lambda$ such that the vanishing subspace is $E_{\lambda}^{\perp}$, so $E_{\lambda}$ is all of $M_{p}$. But then $A$ is $\lambda$ times a complex structure on $M_{p}$. Furthermore, since Hol acts trivially on $A, \lambda^{-1} A$ can be extended to a global integrable complex structure. $M$ is thus a complex symmetric space, and Hol is a subgroup of $U(k)$, with $2 k=n$.

Since $A \in \operatorname{ker} R^{M}$, and since $R^{M}$ is a self-adjoint map from $\mathfrak{s o}(T M)$ to itself, $A$ is orthogonal to the image of $R^{M}$. Thus, for any $B \in \Lambda^{2}(T M), 0=\left\langle A, R^{M}(B)\right\rangle=$ 
$R^{M}(B) \in \mathfrak{u}(k)$ has complex trace 0 , and the holonomy of $M$ lies in $S U(k)$, not just in $U(k)$. The determinant of the tangent bundle (viewed as a complex $k$ dimensional vector bundle) is trivial, with a flat connection. In other words, $M$ is a Calabi-Yau manifold.

Calabi-Yau manifolds have Ricci-flat metrics (see Ch.9, Theorem 4.6 in [K-N]). However, Ricci-flat homogeneous spaces (and in particular Ricci-flat symmetric spaces) are flat (see section 7.61 in [B]). Since $M$ is simply connected, it must equal $\mathbb{C}^{k}$. However, $\mathbb{C}^{k}=\mathbb{R}^{k} \times \mathbb{R}^{k}$ is reducible, and we have a contradiction.

Remark. The simple connectivity assumption in the previous theorem was necessary to ensure that Hol is connected. However, a version of the theorem holds in the non-simply connected case. If $M$ is not simply connected, we work on the universal cover $\tilde{M}$. If $\tilde{M}$ is irreducible, then Condition A holds on $\tilde{M}$, and therefore holds on $M$.

Remark. Condition A is considerably weaker than the nonexistence of a parallel 2 -form, which is a common condition applied to symmetric spaces. We have just seen that a parallel section of $\mathfrak{s o}(T M)$ (or equivalently a parallel 2-form) on an irreducible symmetric space must be a complex structure, but many irreducible symmetric spaces are indeed complex, and their complex structures are indeed parallel. Condition A allows such structures, just not in the kernel of $R^{M}$.

To understand the general case, we will examine first how Condition A behaves under products with irreducible symmetric spaces:

Lemma 4.2. Let $M \neq \mathbb{R}$ be a simply connected irreducible symmetric space and $N$ a simply connected symmetric space, not necessarily irreducible. Then $M \times N$ satisfies condition $A$ if and only if $N$ does.

Proof. The curvature of $M \times N$ is the direct sum of the curvature of $M$ and the curvature of $N$, so the kernel of $R^{M \times N}$ at a point $(p, q)$ is the kernel of $R^{M}$ at $p$ plus the kernel of $R^{N}$ at $q$ plus the linear subspace spanned by the wedge of $M_{p}$ and $N_{q}$. Similarly, the image of $R^{M \times N}$ is the direct sum of the images of $R^{M}$ and $R^{N}$ under the diagonal inclusion $\mathfrak{s o}\left(M_{p}\right) \oplus \mathfrak{s o}\left(N_{q}\right) \subset \mathfrak{s o}\left(M_{p} \oplus N_{q}\right)$.

It is clear then that $\left[\operatorname{ker} R^{M}, \operatorname{Im} R^{M \times N}\right]=\left[\operatorname{ker} R^{M}, \operatorname{Im} R^{M}\right]$ which spans $\operatorname{ker} R^{M}$. Similarly, $\left[\operatorname{ker} R^{N}, \operatorname{Im} R^{M \times N}\right]=\left[\operatorname{ker} R^{N}, \operatorname{Im} R^{N}\right]$. Since $M$ is irreducible and $M \neq$ $\mathbb{R}$, it is easy to see that $\left\{R^{M}\left(\mathfrak{s o}\left(M_{p}\right)\right) M_{p}\right\}$ spans all of $M_{p}$. Thus for any $x \in M_{p}$ we can find $a_{i} \in \mathfrak{s o}\left(M_{p}\right)$ and $x_{i} \in M_{p}$ with $\sum_{i} R^{M}\left(a_{i}\right) x_{i}=x$. This implies that

$$
x \wedge y=\sum_{i} R^{M}\left(a_{i}\right) x_{i} \wedge y=-\sum_{i}\left[x_{i} \wedge y, R^{M}\left(a_{i}\right)\right]
$$

and therefore $\operatorname{span}\left(M_{p} \wedge N_{q}\right)=\operatorname{span}\left(\left[M_{p} \wedge N_{q}, \operatorname{Im} R^{M}\right]\right)$. It follows from this that the span of [ $\left.\operatorname{ker} R^{M \times N}, \operatorname{Im} R^{M \times N}\right]$ equals $\operatorname{ker} R^{M \times N}$ if and only if $\operatorname{span}\left(\left[\operatorname{ker} R^{N}, \operatorname{Im} R^{N}\right]\right)$ equals $\operatorname{ker} R^{N}$, giving us the conclusion of the lemma.

Theorem 1.1. Let $M$ be a simply connected symmetric space with de Rham decomposition given by $M=\mathbb{R}^{n_{0}} \times M_{1} \times \cdots \times M_{l}$. Then $M$ satisfies condition $A$ if and only if $n_{0} \leq 1$. Equivalently, $M$ satisfies condition $A$ if and only if the dimension 
Proof. From the last two lemmas it is clear that $M_{1} \times \cdots \times M_{l}$ satisfies condition A. On the other hand, the condition holds on $\mathbb{R}^{n_{0}}$ precisely when $n_{0} \leq 1$. Thus, the first part of the theorem follows trivially from Lemma 4.2. The statement about the center follows, since the center of $G$ generates the flat factors in the de Rham decomposition.

\section{EXAMPLES AND APPLICATIONS}

\section{A. Bundles over spheres.}

The sphere $S^{n}$ is a symmetric space with $G_{0}=S O(n+1), H_{0}=S O(n), G=$ $\operatorname{Spin}(n+1)$ and $H=\operatorname{Spin}(n)$. By the main theorem, a parallel vector bundle over $S^{n}$ corresponds to a representation of $\operatorname{Spin}(n)$, and is thus the Whitney sum of vector bundles corresponding to irreducible representations of $\operatorname{Spin}(n)$. Our analysis simplifies to listing the irreducible representations of $\operatorname{Spin}(n)$.

If $n=2$, then $S O(2)$ and $\operatorname{Spin}(2)$ are both circle groups. We identify $S O(2)$ with $\mathbb{R} /(2 \pi \mathbb{Z})$ and $\operatorname{Spin}(2)$ with $\mathbb{R} /(4 \pi \mathbb{Z})$, so that the Lie algebras of $S O(2)$ and $\operatorname{Spin}(2)$ are naturally identified. Besides the trivial 1-dimensional representation, the irreducible representations of $\operatorname{Spin}(2)$ are all 2-dimensional, namely

$$
\rho_{k}(x)=\left(\begin{array}{cc}
\cos (k x / 2) & \sin (k x / 2) \\
-\sin (k x / 2) & \cos (k x / 2)
\end{array}\right)
$$

The corresponding vector bundle is a plane bundle of Euler class $k$, or equivalently a complex line bundle of first Chern class $k$. Important special cases are the Hopf fibrations $k= \pm 1$ and the tangent bundle $k=2$.

Before analyzing the higher dimensional cases, we recall some general concepts and facts about orthogonal representations, cf. also [BD]. If $V$ is an inner product space, then an irreducible orthogonal representation $\rho: H \rightarrow S O(V)$ is either unitary for some complex structure, or else its complexification is irreducible, and the latter is of real type; i.e., it admits a real structure, or in other words, a complex conjugate linear map $J: \mathbb{C} \otimes V \rightarrow \mathbb{C} \otimes V$ that squares to the identity and commutes with the action of $H$. Its restriction to the +1 eigenspace of $J$ is the original real representation. A unitary representation is said to be of quaternionic type if it admits a quaternionic structure, or in other words, a complex conjugate linear map that squares to minus the identity and commutes with $H$.

If $n=3$, then $\operatorname{Spin}(3)=S U(2)$, and the irreducible representations are well known, see for example [BD]. The fundamental representation, corresponding to matrix multiplication, has complex dimension 2 , and has a quaternionic structure given by $J\left(z_{1}, z_{2}\right)=\left(-\bar{z}_{2}, \bar{z}_{1}\right)$ for $\left(z_{1}, z_{2}\right) \in \mathbb{C}^{2}$. All other complex irreducible representations are obtained from symmetric products of the fundamental representation. Explicitly, let $V_{k}$ denote the space of homogeneous polynomials $p$ of degree $k$ in two complex variables,

$$
p\left(z_{1}, z_{2}\right)=\sum_{r=0}^{k} a_{r} z_{1}^{r} z_{2}^{k-r},
$$

of complex dimension $k+1$. The action of $S U(2)$ on $V_{k}$ is given by 
In particular, the structure map $J$ on $\mathbb{C}^{2}=V_{1}$ induces a structure map $J$ on $V_{k}$ satisfying $J^{2} p=(-1)^{k} p$. Thus, the odd-dimensional representations have an underlying real structure, while the (complex) even-dimensional representations have a quaternionic structure. Complex irreducible representations of odd dimension $2 \ell+1$ therefore correspond to real irreducible representations of dimension $2 \ell+1$, while complex irreducible representations of dimension $2 \ell$ correspond to real irreducible representations of dimension $4 \ell$. The odd-dimensional irreducible representations also are representations of $S O(3)$ (since $-1 \in S U(2)$ acts trivially on $V_{2 l}$, so that the action of $S U(2)$ induces one of $S O(3)=S U(2) / \pm 1)$, whereas the even-dimensional irreducible representations are not.

Over $S^{3}$, therefore, there exists a unique irreducible parallel unitary bundle in each (complex) dimension. Irreducible Riemannian parallel vector bundles exist in odd dimensions, and in dimensions divisible by 4 . The first few examples are:

1. The trivial bundle, of real dimension 1.

2. The tangent bundle, of dimension 3. This corresponds to the fundamental representation of $S O(3) \cdot \Lambda^{2}(T M)$ is also 3 -dimensional, and is isomorphic to $T M$ via the Hodge star operator.

3. The spinor bundle, of complex dimension 2 and real dimension 4, corresponding to the fundamental representation of $S U(2)$.

4. The quadrupole bundle of traceless symmetric endomorphisms from $T M$ to itself, of real dimension 5 .

If $n=4$, then $\operatorname{Spin}(n)=S U(2) \times S U(2)$. The irreducible representations are tensor products of the representations of the first and second factors. If we take the $k_{1}+1$-dimensional representation of the first factor and the $k_{2}+1$-dimensional representations of the second, we obtain a representation of complex dimension $\left(k_{1}+1\right)\left(k_{2}+1\right)$ with a structure map $J$ that squares to $(-1)^{k_{1}+k_{2}}$. We label this representation $\left(k_{1}, k_{2}\right)$. The complex dimension of $\left(k_{1}, k_{2}\right)$ is always $\left(k_{1}+1\right)\left(k_{2}+1\right)$, while the real dimension is $\left(k_{1}+1\right)\left(k_{2}+1\right)$ if $k_{1}+k_{2}$ is even and $2\left(k_{1}+1\right)\left(k_{2}+1\right)$ if $k_{1}+k_{2}$ is odd. Once again, we see that real irreducible representations come only in odd dimensions and in dimensions that are multiples of 4 .

The first few examples of irreducible parallel Riemannian bundles are:

1. The trivial bundle, of dimension 1 , corresponding to the representation $(0,0)$.

2. The bundle $\Lambda^{+}$of self-dual 2 -forms on $S^{4}$, of real dimension 3 , corresponding to the representation $(2,0)$.

3. The bundle $\Lambda^{-}$of anti-self-dual 2 -forms on $S^{4}$, of real dimension 3 , corresponding to the representation $(0,2)$.

4. The tangent bundle, of real dimension 4, corresponding to the representation $(1,1)$.

5. The bundle $S^{+}$of positive spinors, of complex dimension 2 and real dimension 4 , originating from the representation $(1,0)$. The parallel connection is the standard round self-dual $S U(2)$ instanton of second Chern class -1 . If we identify $S^{4}=$ $\mathbb{H P}^{1}$, this is the tautological quaternionic line bundle, and the unit sphere bundle is a Hopf fibration $S^{7} \rightarrow S^{4}$.

6. The bundle $S^{-}$of negative spinors, corresponding to the representation $(0,1)$. The parallel connection, with structure group $S U(2)$, is the round anti-self-dual 
anti-instanton, of second Chern class 1.

7. There are bundles of real dimension 5, corresponding to the representations $(4,0)$ and $(0,4)$. These are the traceless symmetric endomorphisms of $\Lambda^{+}$and $\Lambda^{-}$, respectively.

Combining these low-dimensional irreducible representations, we obtain a list of all the rank-4 Riemannian vector bundles over $S^{4}$ with parallel curvature.

Proposition 5.1. There are exactly six rank-4 Riemannian vector bundles over $S^{4}$ with parallel curvature. Three of these, namely $S^{+}, S^{-}$and the trivial bundle, admit a complex structure. The corresponding rank-2 complex vector bundles have second Chern class $-1,+1$ and 0 , respectively. The remaining three Riemannian vector bundles, namely $T M, \mathbb{R} \oplus \Lambda^{+}$and $\mathbb{R} \oplus \Lambda^{-}$, are not complex.

Proof. These examples are constructed from representations of Spin(4), and there are no other real 4-dimensional representations. All that remains is to show that these bundles are distinct. This can be seen from their characteristic classes. TM and $S^{ \pm}$all have nontrivial Euler classes ( 2 and $\mp 1$ respectively), and their Pontryagin classes are $0, \pm 2$. The bundles $\mathbb{R}^{4}, \mathbb{R} \oplus \Lambda^{+}$and $\mathbb{R} \oplus \Lambda^{-}$all have trivial Euler class, but their Pontryagin classes are all distinct ( 0 and \pm 4 , respectively).

If $n>4$, then $S O(n)$ is simple, and $\operatorname{spin}(n)$ is a simple Lie Algebra. The smallest nontrivial representation is the fundamental representation of $S O(n)$. Hence there are no nontrivial parallel bundles of dimension less than $n$, and the only nontrivial parallel bundle of dimension $n$ is the tangent bundle. Other noteworthy representations, and their corresponding bundles, are

1 . The $k$-th exterior power of the fundamental representation of $S O(n)$ has dimension $\left(\begin{array}{l}n \\ k\end{array}\right)$, and corresponds to the bundle of $k$-forms on $S^{n}$.

2. The symmetric product of the fundamental representation of $S O(n)$ with itself splits as a trivial factor plus an $[n(n+1)-2] / 2$ irreducible representation. The latter corresponds to the traceless symmetric endomorphisms of $T M$.

3. The fundamental representation of $\operatorname{Spin}(n)$ has complex dimension $2^{n / 2}$ if $n$ is even and $2^{(n+1) / 2}$ if $n$ is odd. If $n$ is even the spin bundle splits as the sum of positive and negative spinor bundles, each of complex dimension $2^{(n-2) / 2}$.

\section{B. Bundles over complex projective spaces.}

The complex projective space $M=\mathbb{C P}^{n}$ is naturally the quotient of $U(n+1)$ by $U(1) \times U(n)$. However, this quotient does not describe the structure of $\mathbb{C P}^{n}$ as a symmetric space, since the center of $U(n+1)$ is contained in $U(1) \times U(n)$. We must take the quotient of both $U(n+1)$ and $U(1) \times U(n)$ by $U(1)$. The question is, which $U(1)$ ?

The most natural choice of $U(1)$ is the center $Z$ of $U(n+1)$. Let $G_{0}=U(n+1) / Z$, and let $H_{0}=(U(1) \times U(n)) / Z \sim U(n) . G_{0} / H_{0}$ is indeed $\mathbb{C P}^{n}, H_{0}$ is the holonomy group of $\mathbb{C P}^{n}$, and $G_{0}$ is the bundle of complex orthonormal frames of $T M$.

$G_{0}$ is not simply connected, however. Its universal cover is $G=S U(n+1)$, and the covering map is $n+1$-to-1. $G$ can be viewed as the quotient of $U(n+1)$ by $Z^{\prime}=$ $U(1) \times I_{n}$, where $I_{n}$ is the $n \times n$ identity matrix, while $H=(U(1) \times U(n)) / Z^{\prime} \sim U(n)$. Although $H$ and $H_{0}$ are both isomorphic to $U(n)$ as groups, $H$ is the $n+1$-fold 
$G$ itself can be viewed as a bundle of frames, just not of $T M$. G is the bundle of complex orthonormal frames of $L \otimes_{\mathbb{C}} T M$, where $L$ is the tautological complex line bundle over $\mathbb{C P}^{n}$. Equivalently, $L \otimes_{\mathbb{C}} T M$ is the orthogonal complement to $L$ in the trivial $\mathbb{C}^{n+1}$ bundle over $\mathbb{C P}^{n}$.

There are infinitely many nontrivial complex 1-dimensional (or real 2-dimensional) representations of $H=U(n)$, namely all powers of the determinant. The determinant representation itself yields a line bundle of Chern class +1 . This is the determinant bundle of $L \otimes_{\mathbb{C}} T M$, and is also isomorphic to $L^{*}$, the complex dual of $L$. The $k$-th power of the determinant yields $\left(L^{*}\right)^{k}$ if $k>0$, or $L^{-k}$ if $k<0$. (For $n=1$, this replicates our previous analysis of $M=S^{2}$.)

Note that the determinant representation of $H_{0}$ yields the determinant of $T M$ (viewed as a complex vector bundle), which has Chern class $n+1$, and all complex line bundles constructed from representations of $H_{0}$ have first Chern class divisible by $n+1$. This demonstrates once again that to get all parallel bundles we must consider all representations of $H$, not just representations of $H_{0}$.

After the trivial bundle, the determinant, and powers of the determinant, the smallest unitary representations of $U(n)$ are the fundamental representation and its dual, both of complex dimension $n$. There are an infinite number of complex representations of this dimension, obtained by multiplying the trivial representation (or its dual) by arbitrary powers of the determinant. The smallest nontrivial real (i.e. not complex) irreducible representation is the adjoint representation, of real dimension $n^{2}-1$. 


\section{Lie Groups.}

Every connected Lie group $K$ which is the product of a compact group and a vector group is a symmetric space. If $K$ is simply connected, we can take $G=$ $K \times K$, and let $H \sim K$ be the diagonal subgroup of $G$. Parallel bundles over $K$ thus correspond to representations of $K$ itself. The tangent bundle of $K$ corresponds to the adjoint representation, as the tangent space of $K$ at the origin is naturally isomorphic to the Lie algebra of $K$. It follows from Corollary 5.3 below that if $K$ has no Euclidean factor, then the unit tangent bundle of $K$ admits a metric of constant positive scalar curvature.

Applying this construction to $K=S U(2)$, we recover our previous results about $M=S^{3}$.

\section{Sphere bundles and constant scalar curvature.}

Let $E$ denote a rank $k$ vector bundle with parallel connection over $M=G / H$. It follows from the main theorem that if $G$ admits a bi-invariant metric, then $E$ and the unit sphere bundle $E^{1}$ of $E$ admit complete metrics of nonnegative sectional curvature: Endow $\mathbb{R}^{k}$ with any $O(k)$ invariant metric. Then the diagonal action of $H$ on the metric product $G \times \mathbb{R}^{k}$ is by isometries, and there is a unique metric on the quotient $E=G \times{ }_{\rho} \mathbb{R}^{k}$ (respectively $G \times{ }_{\rho} S^{k-1}$ ) for which the projection $G \times \mathbb{R}^{k} \rightarrow E$ (resp. $G \times S^{k-1} \rightarrow E^{1}$ ) becomes a Riemannian submersion, see [C]. Moreover, if $H$ acts transitively on the unit sphere in $\mathbb{R}^{k}$, then $E^{1}$ is a homogeneous space under the isometric action $g\left[\left(g^{\prime}, u\right)\right]:=\left[\left(g g^{\prime}, u\right)\right], g \in G$, and in particular, $E^{1}$ has constant scalar curvature. It is noteworthy that there are vector bundles over the sphere that admit nonnegatively curved metrics but no parallel connections: One of these, in fact, is a rank 4 bundle over $S^{4}$ whose unit sphere bundle is a Milnor exotic 7-sphere, see [G-M] and [R].

If $H$ does not act transitively on $S^{k-1}$, then the resulting metric on the unit sphere bundle is not, in general, homogeneous. Nevertheless, when the action of $H$ is irreducible, then there exists a metric of constant scalar curvature on $E^{1}$ : Decompose $T E=\mathcal{H} \oplus \mathcal{V}$ as a sum of subbundles $\mathcal{H}$ (the horizontal distribution defining the connection) and $\mathcal{V}$ (the vertical distribution tangent to the fibers of $\pi: E \rightarrow M$ ) over $E$. The inner product on $\mathcal{H}$ is defined by pulling back the one on $T M$ via $\pi_{*}: \mathcal{H} \rightarrow T M$. The one on $\mathcal{V}$ is induced by the $O(k)$ invariant metric on $\mathbb{R}^{k}$. Finally, declare both distributions to be mutually orthogonal. This defines a connection metric on $E$, and the projection $\pi: E \rightarrow M$ is a Riemannian submersion with totally geodesic fibers. It can be shown $[\mathrm{S}-\mathrm{W}]$ that if the sectional curvature $K_{M}$ of $M$ is strictly positive (i.e., if $M$ is a compact rank one symmetric space), then $K_{E} \geq 0$, provided the fiber metric is sufficiently curved.

Proposition 5.2. The connection metric on E induces a constant scalar curvature metric on the unit sphere bundle $E^{1}$.

Proof. Let $s_{E}, s_{M}$, and $s_{F}$ denote the scalar curvatures of $E, M$, and the fiber $F$ respectively. Since $\pi: E \rightarrow M$ has totally geodesic fibers,

$$
s_{E}=s_{m} \circ \pi+s_{F}-|A|^{2}, \quad|A|^{2}:=\sum_{i, j}\left|A_{x_{i}} x_{j}\right|^{2},
$$

cf. [B, 9.37]. Here $A$ denotes the O'Neill tensor of $\pi, A_{X} Y=\frac{1}{2}[X, Y]^{v}$ for horizontal 
other hand, for unit $u \in E$ and $r>0$,

$$
|A|^{2}(r u)=\frac{1}{4} G^{2}(r) \sum_{i, j}\left|R^{E}\left(x_{i}, x_{j}\right) u\right|^{2},
$$

where $d r^{2}+G^{2}(r) d \sigma^{2}$ is the fiber metric in polar coordinates, see [S-W]. It therefore suffices to check that the map $C: E^{1} \rightarrow \mathbb{R}^{+}$given by $C(u)=\sum\left|R\left(x_{i}, x_{j}\right) u\right|^{2}$ is constant. Observe that $C(u)=\langle\tilde{C} u, u\rangle$, where $\tilde{C}: E \rightarrow E$ is defined as

$$
\tilde{C}=-\sum_{i, j} R^{E}\left(x_{i}, x_{j}\right) \circ R^{E}\left(x_{i}, x_{j}\right)
$$

But $\tilde{C}$ is parallel since $R^{E}$ is, and therefore commutes with the action of the holonomy group of $E$. The latter is by assumption irreducible, so that by Schur's lemma, $\tilde{C}$ is a multiple of the identity. This implies that $C$ is constant as claimed.

Corollary 5.3. Let $M$ be an irreducible symmetric space with nonnegative sectional curvature. Then the unit tangent bundle of $M$ admits a metric of nonnegative sectional curvature and constant scalar curvature.

Proof. The first part of the statement is essentially due to Cheeger [C]. The claim about the scalar curvature is an immediate consequence of Proposition 5.2 together with the irreducibility assumption.

Acknowledgements. We thank Sharad Agnihotri and Jan Segert for helpful discussions. The work of L.S. is partially supported by NSF grant DMS-9626698.

\section{REFERENCES}

[B] A. Besse, Einstein manifolds, Springer-Verlag, 1987.

[B-L] J.P. Bourguignon \& B.H. Lawson, Stability and isolation phenomena for Yang-Mills fields, Commun. Math. Phys. 79 (1981), 189-230.

[B-H] D. Brandt \& J.C. Hausmann, Théorie de jauge et symmétries des fibrés, Ann. Inst. Fourier, Grenoble 43 (1993), 509-537.

[B-D] T. Bröcker \& T. t. Dieck, Representations of compact Lie groups, GTM Vol 98, SpringerVerlag, 1985.

[C] J. Cheeger, Some examples of manifolds of nonnegative curvature, J. Diff. Geom. 8 (1972), 623-628.

[D-K] S. Donaldson \& P. Kronheimer, The geometry of four-manifolds, Oxford University Press, 1991.

[G-M] D. Gromoll \& W. Meyer, An exotic sphere with nonnegative sectional curvature, Ann. Math. 100 (1974), 401-406.

[K-N] S. Kobayashi \& K. Nomizu, Foundations of differential geometry, Interscience, 1969.

[P] W. Poor, Differential geometric structures, McGraw-Hill, 1981.

[R] A. Rigas, Some bundles of nonnegative curvature, Math. Ann. 232 (1978), 187-193.

[S-W] M. Strake \& G. Walschap, Connection metrics of nonnegative curvature on vector bundles, Manuscripta Math. 66 (1990), 309-318.

[W] H.C. Wang, On invariant connections over a principal fiber bundle, Nagoya Math. J. 13 (1958), 1-19. 
E-mail address: guijarro@math.upenn.edu

University of Texas, Austin, TX 78712

E-mail address: sadun@math.utexas.edu

University of Oklahoma, Norman, OK 73019

E-mail address: gwalschap@math.ou.edu 